\title{
Niveau d'infestation des arbres fruitiers des groupements végétaux par Phragmanthera capitata (Sprengel) S. Balle (Loranthaceae) dans la région littorale du Cameroun
}

\author{
Didier Siegfried DIBONG ${ }^{1}{ }^{*}$, Nestor Laurier ENGONE OBIANG ${ }^{2}$, Ndongo DIN $^{1}$, \\ Richard Jules PRISO ${ }^{1}$, Désiré Victor TAFFOUO ${ }^{1}$, Henri FANKEM ${ }^{1}$, Georges SALLE ${ }^{3}$ \\ et AMOUGOU AKOA ${ }^{4}$ \\ ${ }^{1}$ Laboratoire d'Ecologie végétale, Département de Biologie des Organismes végétaux, \\ Faculté des Sciences, B. P. 24157, Université de Douala, Cameroun. \\ ${ }^{2}$ Institut de Recherche d'Ecologie Tropicale (IRET), BP 13354 Libreville, Gabon. \\ ${ }^{3}$ Laboratoire de Parasitologie végétale, Université Pierre et Marie Curie (Paris VI), 4 place \\ Jussieu, case courrier 155, 75252 Paris Cedex 05, France. \\ ${ }^{4}$ Département de Biologie et Physiologie Végétales, B. P. 812, Université de Yaoundé I, Cameroun. \\ *Auteur correspondant,E-mail : didierdibong @yahoo.fr
}

\section{RESUME}

$\mathrm{Au}$ Cameroun, Phragmanthera capitata parasite de nombreuses essences ligneuses sauvages et cultivées, et est responsable de la réduction de leur rendement. L'optique de cette étude est de justifier le comportement de cette Loranthaceae ubiquiste dans les groupements végétaux homogènes et hétérogènes des sites retenus du littoral camerounais. Les groupements végétaux homogènes sont des plantations agricoles constituées de Hevea brasiliensis et appartenant à la société Hévéa du Cameroun (Hévécam) située à Nyétté. Les groupements végétaux hétérogènes appartiennent à quatre sites de la région de Douala : un verger à Makondo et une plantation agricole paysanne à Cola acuminata à Penja, deux jardins de cases (Logbessou et axe routier aéroport-Bonanjo). Sur chaque arbre hôte ont été notés: le diamètre du tronc à $1,30 \mathrm{~m}$ du sol, les différentes espèces de Loranthaceae reconnues et le nombre de touffes comptées. Dans les groupements végétaux hétérogènes, 16 espèces hôtes réparties dans 12 genres et 10 familles sont parasitées par $P$. capitata. Cette dernière est la plus fréquente et la plus abondante de toutes les Loranthaceae et sa dissémination est facilitée par des facteurs d'origine anthropique. Il est indispensable et urgent de mettre en place un programme de recherche de lutte ciblée pour les plantes fruitières et ornementales.

(C) 2009 International Formulae Group. All rights reserved.

Mots-clés : Phragmanthera capitata, Loranthaceae, dissémination, espèces hôtes, lutte ciblée.

\section{INTRODUCTION}

Les Loranthaceae sont des arbrisseaux épiphytes, chlorophylliens, qui vivent en hémiparasites sur les branches d'arbres et d'arbustes, sauvages ou cultivés (Balle (1982). Ces plantes qui se présentent sous forme de touffes, sont ancrées dans le bois de l'hôte grâce à un suçoir qui permet d'établir des liaisons fonctionnelles avec l'appareil conducteur de l'hôte (Sallé et al., 1998). Le parasite prélève ainsi l'eau, les sels minéraux et le complément de matière organique dont il a besoin.

Les Loranthaceae appartiennent aux Angiospermes parasites qui ne représentent que $2 \%$ de l'ensemble des plantes à graines (Parker et Riches, 1993; Raynal-Roques et Paré, 1998). Mais dans certaines conditions, elles peuvent devenir de véritables fléaux.

Phragmanthera capitata, est largement répandue en Afrique, notamment au Cameroun, au Nigéria, au Gabon et en Côte 
d'Ivoire (Gill et Onybe, 1990; Sallé, 1994; Hévécam, 1995; Engone et al., 2005; Engone et Sallé, 2006). Les cultures de rente telles que l'hévéa, l'avocatier, le cacaoyer, le caféier, les agrumes apparaissent fortement parasitées. Aujourd'hui, la suppression mécanique des branches parasitées de l'hôte s'avère illusoire. Il est donc indispensable d'entreprendre une étude des facteurs écologiques qui président à l'expansion de $P$. capitata dans le littoral camerounais. En effet, la notion de lutte ciblée s'impose pour les pays en voie de développement, essentiellement agricoles. De plus, le réchauffement planétaire devenu une réalité accroît les effets négatifs de $P$. capitata considérée désormais comme un véritable fléau agronomique.

La présente étude a été entreprise dans l'optique de justifier le comportement de $P$. capitata dans les groupements végétaux homogènes et hétérogènes des sites retenus du littoral camerounais.

\section{MATERIEL ET METHODES Sites d'étude}

Les travaux dont les résultats sont restitués dans cette publication se sont déroulés dans deux régions : Nyétté et Douala.

\section{Région de Nyétté}

Les sites d'étude des groupements végétaux homogènes sont localisés à Nyétté située à $02^{\circ} 45^{\prime} \mathrm{N}$ de latitude, $10^{\circ} 03^{\prime} \mathrm{E}$ de longitude et $20 \mathrm{~m}$ d'altitude. Le climat de la région est bimodal, marqué par la mousson en deuxième saison. La saison des pluies (environ 2195,9 mm par an) va de février à novembre. La température moyenne annuelle est élevée et constante $\left(27,3^{\circ} \mathrm{C}\right)$.

La moyenne du minimum de température calculée à Nyétté pour 30 années $\left(1976\right.$ - 2003) est de $23,4{ }^{\circ} \mathrm{C}$ en septembre et la moyenne du maximum de $26,7{ }^{\circ} \mathrm{C}$ en février. L'humidité relative de l'air reste élevée toute l'année avec une moyenne de $82,6 \%$. La plus petite valeur est environ de $60 \%$ en février et la plus grande de $100 \%$ en saison de pluies (Hévécam, 2006).

\section{Région de Douala}

Douala (latitude, $03^{\circ} 40-04^{\circ} 11^{\prime} \mathrm{N}$; longitude, $09^{\circ} 16^{\prime}-09^{\circ} 52^{\prime} \mathrm{E}$; altitude, $13 \mathrm{~m}$ ) a un climat qui appartient au domaine équatorial d'un type particulier dit
« camerounien» qui se caractérise par deux saisons avec une longue saison de pluies (au moins 9 mois), des précipitations abondantes (environ $4000 \mathrm{~mm}$ par an), des températures élevées $\left(26,7{ }^{\circ} \mathrm{C}\right)$ et stables. La moyenne minimale de température à Douala pour 30 années (1961-1990) est de $22,6{ }^{\circ} \mathrm{C}$ en juillet et la moyenne maximale de température de 32,3 ${ }^{\circ} \mathrm{C}$ en février. L'humidité relative de l'air reste élevée et constante toute l'année et avoisine 100\% (Din et al., 2008).

\section{Echantillonnage des groupements végétaux dans les agrosystèmes}

Dans les paysages anthropisés, la caractérisation des Loranthaceae a porté sur les groupements végétaux homogènes et hétérogènes. Les travaux se sont déroulés de janvier 2007 à juin 2008 selon les exigences de notre protocole expérimental.

Les groupements végétaux homogènes étudiés sont des plantations de Hevea brasiliensis appartenant à la société Hévéa du Cameroun (Hévécam). Ces plantations sont localisées à Nyétté, située à $35 \mathrm{~km}$ environs au nord-est de Kribi ; elles sont divisées en plusieurs secteurs séparés par des marécages. Chaque secteur est représenté par un village, abrégé par la lettre $\mathrm{V}$ suivi d'un numéro d'identification, par exemple V 14. Chaque secteur est composé d'un nombre variable de blocs, désignés par les exploitants par deux nombres a et $b$, sous la forme $a / b$, suivie d'une lettre en majuscule de l'alphabet français (par exemple, 20/15 C).

Les exploitants appellent bloc, tout territoire de surface variable uniquement planté d'arbres appartenant à un même clone. Les arbres sont disposés en ligne et le nombre de lignes est variable d'un bloc à l'autre. En revanche, les distances entre les lignes et entre les arbres disposés sur une même ligne sont constantes, respectivement $8 \mathrm{~m}$ et $2,25 \mathrm{~m}$. Chaque clone est identifié par un sigle de deux ou trois lettres indiquant son origine et par un numéro d'ordre. Le clone GT 1 (Gondang Tapen) est originaire d'Indonésie (Delabarre et Serier, 1995).

Dans les plantations, les différents clones ne sont pas répartis de façon équivalente. Le clone GT 1 est de loin le plus répandu et justifie le choix opéré pour notre étude. Tous les clones sont obtenus par 
multiplication végétative (greffage) : en sacs, en «stumps » et en «planting».

Afin d'estimer le niveau de parasitisme des hévéas, dix relevés ont été réalisés dans différents blocs des plantations industrielles, répartis dans cinq villages. Les plantations villageoises ont été évitées et une surface d'échantillonnage de 20 ha a été retenue pour chaque bloc. Les blocs choisis datent de 1984 et se répartissent comme suit, en fonction des villages :

- village V 10 : bloc 9 /19 D ;

- village V 12 : blocs 20/15 C, 17/12 D, 13/15

$\mathrm{D}$;

- village V 13 : blocs 11/12 C, 11/11 D ;

- village V 14 : blocs $12 / 22$ C, 12/21 C, 11/20

D ;

- village V 18 : bloc16/17 D.

Les plantations industrielles bordent la forêt voisine. Le choix des blocs a été orienté, de façon à clarifier l'origine des Loranthaceae.

Au contraire des groupements végétaux homogènes, les groupements végétaux hétérogènes présentent un nombre variable d'espèces hôtes selon les sites. Quatre sites de la région de Douala ont été prospectés :

- un verger à Makondo et une plantation agricole paysanne à Cola acuminata, à Penja, dans lesquels la surface échantillonnée est 0,5 ha pour chaque site ;

- deux jardins de cases (Logbessou et axe routier Aéroport-Bonanjo) où tour à tour, une surface de $10.000 \mathrm{~m}^{2}$ (2000 m x $\left.50 \mathrm{~m}\right)$ a été délimitée.

Les Loranthaceae étaient identifiées sur les essences ligneuses de diamètre (DBH) supérieur ou égal à $5 \mathrm{~cm}$, pour les groupements végétaux hétérogènes. Dans le groupement homogène, les hévéas sont uniformes et leur diamètre moyen est de 35 $\mathrm{cm}$. L'identification est suivie de l'estimation du parasitisme sur chaque arbre parasité, en comptant le nombre de touffes de $P$. capitata. Cette tâche a été facilitée par les étudiants et les employés de la société Hévéa du Cameroun (Hévécam). Pour chaque site prospecté, le pourcentage de parasitisme $(\mathrm{Pp})$ est donné par la relation suivante :

$\mathrm{Pp}=$ nombre d'arbres parasités/nombre d'arbres total x 100 .

Le logiciel statistica 5.0 a permis de faire le test ANOVA.

\section{RESULTATS}

\section{Les groupements végétaux homogènes}

$P$. capitata est la seule Loranthaceae qui infeste les individus hôtes de Hevea brasiliensis dans le secteur choisi pour le travail. Le pourcentage moyen du parasitisme dans les parcelles est de 63,64\%. Le maximum de parasitisme se situe dans le bloc 12/22 C (V 14) et le minimum dans le bloc 16/17 D (V 18) (Tableau 1).

Origine du parasitisme de Phragmanthera capitata dans les plantations d'hévéa de Nyétté

Pour confirmer l'origine forestière des Loranthaceae et leur préférence pour le bord des cours d'eau, nous avons compilé les résultats des inventaires de Nyétté en trois zones (Tableau 1): proximité de la forêt, bord du fleuve Nyétté à l'intérieur des plantations et intérieur des plantations. Il apparaît que le pourcentage de parasitisme moyen à proximité de la forêt est le plus élevé (>75\%). A l'intérieur des plantations, il devient bas $(<35 \%)$. Cependant, le bord du fleuve Nyétté à l'intérieur des plantations est également favorable à l'installation de Phragmanthera capitata. Ce constat se justifie par le relèvement du pourcentage de parasitisme moyen dans cette zone (> 50\%).

De même, le nombre moyen de touffes de $P$. capitata par arbre hôte a varié par zone. Ainsi, le nombre moyen de touffes par arbre hôte à proximité de la forêt est de 3,56. Il diminue respectivement au bord du fleuve à l'intérieur des plantations $(2,46)$ et davantage à l'intérieur des plantations seulement $(1,92)$. Le nombre de touffes de $P$. capitata varie dans le même sens que le pourcentage de parasitisme moyen dans les trois zones des plantations d'hévéas.

\section{Les groupements végétaux hétérogènes}

Quatre groupements hétérogènes ont été recensés et ils appartiennent chacun, à un site étudié (Tableau 2). Les groupements végétaux hétérogènes les plus diversifiés sont Makondo et Penja avec six espèces hôtes. Les deux autres sites, aéroport-Bonanjo et Logbessou ont chacun, quatre espèces hôtes.

Les trois sites, Makondo, Logbessou et Penja sont riches en arbres fruitiers consommés. Ils sont situés à la périphérie de la zone urbaine. Le site aéroport-Bonanjo 
Tableau 1: Inventaire de Phragmanthera capitata sur les arbres hôtes des clones GT 1, des blocs de 20 ha chacun, des plantations d'hévéa à Nyétté.

\begin{tabular}{|l|l|l|c|c|c|c|c|}
\hline Villages & Parcelles & \multicolumn{1}{|c|}{ Situation } & N.I.R & N.I.P. & N.T.P. & N.P. & N.M.T.A. \\
\hline V14 & $12 / 22 \mathrm{C}$ & $\begin{array}{l}\text { Proximité de } \\
\text { la forêt }\end{array}$ & 658 & 621 & 2916 & 94,38 & 4,43 \\
\hline V14 & $12 / 21 \mathrm{C}$ & $\begin{array}{l}\text { Proximité de } \\
\text { la forêt }\end{array}$ & 508 & 421 & 1387 & 82,87 & 2,73 \\
\hline V12 & $20 / 15 \mathrm{C}$ & $\begin{array}{l}\text { Proximité de } \\
\text { la forêt }\end{array}$ & 643 & 482 & 1776 & 74,96 & 2,76 \\
\hline V14 & $11 / 20 \mathrm{D}$ & $\begin{array}{l}\text { Proximité de } \\
\text { la forêt }\end{array}$ & 636 & 474 & 1282 & 74,53 & 2,01 \\
\hline V10 & $9 / 19 \mathrm{D}$ & $\begin{array}{l}\text { Proximité de } \\
\text { la forêt }\end{array}$ & 851 & 628 & 2216 & 74,79 & 2,60 \\
\hline V13 & $11 / 12 \mathrm{~A}$ & $\begin{array}{l}\text { Intérieur des } \\
\text { plantations } \\
\text { proche des } \\
\text { marécages }\end{array}$ & 514 & 297 & 665 & 57,78 & 1,29 \\
\hline V12 & $17 / 12 \mathrm{D}$ & $\begin{array}{l}\text { Intérieur des } \\
\text { plantations } \\
\text { proche des } \\
\text { marécages } \\
\text { forêt }\end{array}$ & 795 & 452 & 1387 & 56,85 & 1,74 \\
\hline V12 & $13 / 15 \mathrm{D}$ & $\begin{array}{l}\text { Intérieur des } \\
\text { plantations } \\
\text { proche des } \\
\text { marécages }\end{array}$ & 668 & 335 & 891 & 50,15 & 1,33 \\
\hline V13 & $11 / 11 \mathrm{D}$ & $\begin{array}{l}\text { Intérieur des } \\
\text { plantations }\end{array}$ & 404 & 155 & 265 & 38,37 & 0,65 \\
\hline V18 & $16 / 17 \mathrm{D}$ & $\begin{array}{l}\text { Intérieur des } \\
\text { plantations }\end{array}$ & 787 & 250 & 513 & 31,76 & 0,65 \\
\hline Total & & 6464 & 4115 & & & & \\
\hline
\end{tabular}

N.I.R. : Nombre d'individus recensés, N.I.P. : Nombre d'individus parasités, N.T.P. : Nombre de touffes de parasites, N.P. : Pourcentage de parasitisme, N.M.T.A. : Nombre moyen des touffes par arbre

est spécifique par sa richesse en espèces exotiques (Cassia spp., Delonix regia, Persea americana et Terminalia mantaly) de gros diamètres, supérieur ou égal à $80 \mathrm{~cm}$. Les pieds de Terminalia mantaly par leur port étalé, possèdent un nombre de touffes de parasite très élevé. Certains pieds de cette espèce peuvent en compter une soixantaine sur les branches et ces touffes très développées, ont des suçoirs énormes pouvant atteindre $12 \mathrm{~kg}$ environ. L'alignement des individus parasités de cette espèce le long de la route de cet axe est spectaculaire et rappelle le caractère hasardeux des espèces choisies pour le reboisement dans la région.

Le taux du parasitisme dû à Phragmanthera capitata oscille entre 17,53 (Logbessou) et $35,05 \%$ (Penja) dans les quatre sites. Les différences du parasitisme entre les sites sont significatives ( $\mathrm{F}$ calculé $=5,43>\mathrm{F}$ critique $=2,60$, pour $\mathrm{p}<0,05)$. La moyenne du parasitisme par $P$. capitata est de $25 \%$. Huit familles de plantes hôtes de $P$. capitata ont été recensées dans les sites étudiés. Il s'agit des Anacardiaceae, Combretaceae, Lauraceae, Mimosaceae, Myrtaceae, Sterculiaceae, Rubiaceae, Rutaceae. Les Mimosaceae et Sterculiaceae sont les plus diversifiées avec chacune deux espèces parasitées. La famille la plus parasitée est celle des Sterculiaceae avec un taux de parasitisme de $26,60 \%$ suivie de celle des Lauraceae, 24,95\%. La seule Lauraceae présente est Persea americana avec un parasitisme de $24,95 \%$.

Tous les avocatiers rencontrés sont infestés par $P$. capitata. Logbessou est le site qui possède plus de la moitié de l'ensemble des avocatiers recensés (Tableau 2).

Deux autres familles montrent un pourcentage de parasitisme supérieur à $10 \%$. Ce sont les Combretaceae $(18,35 \%)$ et les Anacardiaceae (12,78\%). Spondias monbin est la seule Anacardiaceae parasitée présente sur trois sites: aéroport-Bonanjo, Logbessou 
Tableau 2: Inventaire de Phragmanthera capitata sur les arbres hôtes des groupements végétaux hétérogènes des sites étudiés dans la région de Douala.

\begin{tabular}{|c|c|c|c|c|c|c|c|}
\hline $\begin{array}{l}\text { Familles } \\
\text { hôtes }\end{array}$ & $\begin{array}{c}\text { Espèces } \\
\text { hôtes } \\
\text { parasitées }\end{array}$ & Logbessou & Penja & $\begin{array}{l}\text { Aéroport- } \\
\text { Bonanjo }\end{array}$ & Makondo & Total & $\begin{array}{c}\text { Moyenne } \\
(\%)\end{array}$ \\
\hline \multirow[t]{2}{*}{ Sterculiaceae } & $\begin{array}{l}\text { Theobroma } \\
\text { cacao }\end{array}$ & & 33 & & 3 & 129 & 26,60 \\
\hline & $\begin{array}{l}\text { Cola } \\
\text { acuminata }\end{array}$ & & 93 & & & & \\
\hline Lauraceae & $\begin{array}{l}\text { Persea } \\
\text { americana }\end{array}$ & 64 & 25 & 1 & 31 & 121 & 24,95 \\
\hline Combretaceae & $\begin{array}{l}\text { Terminalia } \\
\text { mantaly }\end{array}$ & 2 & & 78 & 9 & 89 & 18,35 \\
\hline Rutaceae & Citrus spp. & 1 & 8 & & 27 & 36 & 7,42 \\
\hline Anacardiaceae & $\begin{array}{l}\text { Spondias } \\
\text { monbin }\end{array}$ & 15 & & & 47 & 62 & 12,78 \\
\hline \multirow[t]{2}{*}{ Mimosaceae } & Cassia spp. & & & 13 & & 31 & 6,39 \\
\hline & $\begin{array}{l}\text { Delonix } \\
\text { regia }\end{array}$ & & & 18 & & & \\
\hline Myrtaceae & $\begin{array}{l}\text { Psidium } \\
\text { guajava }\end{array}$ & 3 & 6 & & 3 & 12 & 2,48 \\
\hline Rubiaceae & $\begin{array}{l}\text { Coffea } \\
\text { robusta }\end{array}$ & & 5 & & & 5 & 1,03 \\
\hline Total & & 85 & 170 & 110 & 120 & 485 & 100 \\
\hline Moyenne & & 17,53 & 35,05 & 22,68 & 24,74 & 100 & \\
\hline
\end{tabular}

Les valeurs inscrites dans les cellules représentent le nombre d'individus parasités par espèce et par localité.

et Makondo. C'est également la seule espèce fruitière indigène de la forêt littorale originelle. Les autres espèces hôtes parasitées sont exotiques. Spondias monbin est conservée dans les jardins de cases et les plantations agricoles paysannes pour ses fruits très appréciés des populations locales. Tous les individus de cette espèce hôte sont parasités.

Deux familles de plantes hôtes ont un parasitisme supérieur à $5 \%$ et inférieur à $10 \%$ : les Rutaceae $(7,42 \%)$ et les Mimosaceae (6,39\%). Le reste montre un taux d'infestation inférieur à 5\%: les Myrtaceae $(2,48 \%)$ et les Rubiaceae $(1,03 \%)$.

Makondo (située à une vingtaine de kilomètres d'Edéa, chef-lieu du Département de la Sanaga-maritime) constitue un village où $P$. capitata a été observée sur trois manguiers de grands diamètres situés à la gare ferroviaire.

\section{DISCUSSION}

Passage progressif de Phragmanthera capitata de la forêt aux milieux anthropisés
De façon générale, Phragmanthera capitata est plus distribuée dans les sites étudiés que les autres Loranthaceae qui pour la plupart ne parasitent leurs hôtes que dans une partie de cette aire que nous avons explorée.

A l'origine, toutes les Loranthaceae citées existent et se trouvent dans la forêt avant que certaines s'adaptent aux milieux modifiés par l'homme et aux espèces ligneuses hôtes cultivées ou spontanées (Dibong et al., 2008). Le passage de la forêt aux milieux modifiés par l'homme s'est opéré sur place via les disséminateurs.

Au départ, certains individus de $P$. capitata ont pu s'adapter à des espèces hôtes nouvelles pour eux, mais qui leur assurent une nutrition facile et abondante. C'est le cas de Hevea brasiliensis. Puis l'adaptation aux espèces hôtes cultivées ou spontanées s'est répandue dans son aire de parasitisme aux conditions écologiques appropriées. Enfin, $P$. capitata parfaitement adaptée à sa nouvelle niche écologique s'affranchit de la forêt environnante des plantations d'hévéas de Nyétté. Elle peut alors s'étendre à diverses 
espèces hôtes cultivées ou spontanées qu'elle parasite dans divers milieux anthropisés (jardins de cases, vergers, pistes et routes, concessions villageoises, plantations agricoles paysannes ou industrielles).

Toutes les espèces de Loranthaceae sont issues de la forêt et se sont adaptées à l'environnement modifié par l'homme. Le passage de la forêt aux arbres cultivés ou spontanés s'est opéré de manière progressive, à la faveur des actions anthropiques et des habitudes alimentaires. Spondias monbin est un arbre sauvage souvent conservé dans les champs pour ses fruits très succulents, par la tribu «Bassa». Les individus de cette espèce ligneuse par leur grande taille, constituent de bonnes perches de nutrition pour les disséminateurs (Aukema et Martinez del Rio, 2002). De plus les arbres accumulent le parasitisme en rapport avec leur vieillissement (Overton, 1994). L'installation des Loranthaceae est donc facilitée par les arbres laissés sur pied, après destruction de la forêt. Parfois la spécificité de l'hôte intervient dans la distribution du parasite. Les «Bassa» cultivent le safoutier et la plante est devenue subspontanée dans les forêts secondarisées. Tapinanthus ogowensis est une Loranthaceae spécifique de cet hôte qu'elle parasite exclusivement dans le groupement végétal hétérogène de Logbessou. C'est également le cas de Helixanthera mannii qui parasite surtout les cacaoyers et caféiers qui leur assurent une nutrition facile et abondante. Cette adaptation ponctuelle de la forêt à la plantation à Cola acuminata à Penja se fait également avec Persea americana et Citrus spp. dans cette aire. Progressivement, à la faveur de la lutte curative mal pratiquée, les autres Loranthaceae peu abondantes pour l'heure, acquièrent leur ubiquité. En effet, la technique qui consiste à couper les branches d'arbres portant les Loranthaceae à l'aide d'une machette ou d'un échenilloir a ses limites.

Nous pouvons donc supposer que le parasitisme par les Loranthaceae est apparu progressivement et que les différentes espèces de Loranthaceae se sont introduites fortuitement dans les milieux anthropisés et ont colonisé les espèces ligneuses hôtes cultivées ou spontanées. Les autres Loranthaceae moins abondantes seraient de nouvelles venues et il y a lieu de redouter leur éventuelle expansion dans la région littorale voire dans l'ensemble du territoire camerounais. Tel est le cas de Tapinanthus ogowensis et Helixanthera mannii susceptibles de devenir, dans un avenir proche, des fléaux majeurs comme l'est déjà $P$. capitata.

Parasitisme de Phragmanthera capitata dans les groupements végétaux homogènes et hétérogènes

Les plantations d'hévéas ont actuellement atteint un niveau d'infestation élevé. Phragmanthera capitata est largement distribuée sur les hévéas. Mais également sur d'autres espèces ligneuses sauvages ou cultivées (fruitières ou ornementales) de la région littorale. Il n'existe pas de clones d'hévéas résistants à $P$. capitata. Les données du parasitisme moyen des différents clones sont: GT 1, 63\%; PR 107, 10\%; PB 235, 9,5\%; PB 260, 5,1\%; PR 261, 3\%; PB 86, 2,6\%; Avros 2037, 1,2\% (Hévécam, 1995). Le clone GT 1 le plus représenté dans toutes les parcelles des plantations d'hévéas (Engone et al., 2006), montre un degré de parasitisme très élevé. Ce clone est également le plus adapté à la production du latex en Afrique centrale (C. A. T. H., 1998).

Le niveau de parasitisme est aussi élevé dans tous les groupements végétaux hétérogènes. D'autres inventaires liés au parasitisme de $P$. capitata ont donné des pourcentages de parasitisme supérieur ou égal à $60 \%$ au Gabon (Engone et Sallé, 2006).

Les observations ont montré que la présence de $P$. capitata peut exclure la fixation d'une autre Loranthaceae sur le même hôte. De même, l'abondance relative de $P$. capitata sur un hôte particulier ou un site, peut exclure la présence d'une autre Loranthaceae sur ce site: c'est le cas des plantations d'hévéas. Réciproquement, la présence d'une autre Loranthaceae sur un hôte peut exclure celle de $P$. capitata. C'est le cas de Tapinanthus ogowensis sur Dacryodes edulis dont les individus représentent $2 / 3$ des arbres fruitiers cultivés recensés sur le plateau de Logbessou. Sur ce site, cette espèce hôte n'est parasitée que par T. ogowensis, alors que le tiers d'arbres restant est parasité par $P$. capitata. L'existence de cette compétition est liée aux facteurs écologiques ou/et génétiques responsables des sécrétions chimiques 
répulsives (Hoffmann et al., 1986; Hawksworth et Wiens, 1996).

Phragmanthera capitata est très dévastatrice (Dibong et al., 2008). Son ubiquité est remarquable et convient à toutes les variations écologiques du Cameroun (Sonké et al., 2000) et du Gabon (Engone et al., 2006). Très envahissante sur les hôtes parasités, elle a un spectre d'hôtes très large par rapport aux autres Loranthaceae. Elle infeste tous les hôtes parasités identifiés à l'exception de D. edulis, Citrus maxima et $C$. sinensis. Son pourcentage de parasitisme (76,14\%) justifie l'ampleur des dégâts de plus en plus considérables de cet hémiparasite dans la sous-région. Aujourd'hui, suite à l'évolution des mauvaises conditions climatiques, $P$. capitata est devenue un fléau réel contre lequel une lutte énergique est indispensable. Pourtant, les paysans ne semblent pas très avertis et préoccupés par la présence de cette Loranthaceae qui cause des dégâts importants sur les récoltes.

L'espèce hôte la plus parasitée par $P$. capitata est Persea americana. Le parasitisme de l'avocatier est plus élevé dans la région de Yaoundé (centre du Cameroun) et varie de 43,30 à 69,44\% (Sonké et al., 2000). Les fruits riches en vitamines (A, B, C, et $\mathrm{E})$, sels minéraux (fer, magnésium et potassium) et protéines sont très appréciés par les populations concernées. Avec une production annuelle de 1,78 Mt, l'avocatier occupe la sixième place parmi les cultures fruitières tropicales (Zentmyer, 1994). Le Cameroun serait le douzième producteur mondial d'avocats avec 33000 t/an. Ce rendement désormais soumis à la baisse est davantage limité par l'action de divers ravageurs et maladies fongiques. Ainsi, Phytophtora cinnamomi détruit les racines de la plante, alors que Cercospora purpurea est à l'origine de crevasses et pustules sur les fruits (Schall, 1987).

\section{Conclusion}

La Loranthaceae la plus abondante et la plus virulente dans la région littorale est Phragmanthera capitata. L'extension de cette Loranthaceae est favorisée par la fragmentation prononcée de la forêt littorale très anthropisée dont proviendraient les hémiparasites et les habitudes culturelles des tribus concernées. En effet, la domestication de certains ligneux tel que Spondias mangifera produisant des fruits très appréciés pour la consommation locale a été observée à Logbessou et à Makondo chez les «Bassa ». $\mathrm{Ce}$ parasitisme semble renforcé par la variabilité des conditions écologiques et le caractère polyphyte de Phragmanthera capitata qui est devenue une véritable peste végétale même dans les plantations agroindustrielles de Hevea brasiliensis de la société Hévéa du Cameroun (Hévécam). Dans le contexte actuel où l'agriculture constitue le socle de l'économie du pays, il est indispensable et urgent de mettre en place un programme de recherche de lutte ciblée.

\section{REFERENCES}

Aukema J, Martinez del Rio C. 2002. Where does a fruit-eating bird deposit mistletoe seeds? Seed deposition patterns and an experiment. Ecology, 83: 3489-3496.

Balle S. 1982. Loranthacées. In Flore $d u$ Cameroun (vol. 23), Satabié B, Leroy JF (Eds). Yaoundé, Cameroun; 82 p.

C.A.T.H. 1998. Rapport d'activité du quatrième trimestre, $49 \mathrm{p}$.

Delabarre MA, Serier JB. 1995. L'Hévéa. Maisonneuve et Larose; 238 p.

Dibong SD, Din N, Priso RJ, Taffouo VD, Fankem H, Sallé G, Amougou A. 2008. Parasitism of host trees by the Loranthaceae in region of Douala (Cameroon). African Journal of Environmental Science and Technology, 2(11): 371-378.

Din N, Saenger P, Priso JR, Dibong SD, Amougou A. 2008. Logging activities in mangrove forests: A case study of Douala Cameroon. African Journal of Environmental Science and Technology, 2(2): 22-30.

Engone Obiang NL, Paré J, Duredon J, Sallé G. 2005. Germination et développement de la plantule de Helixanthera mannii (Oliv.) Danser (Loranthaceae) sur cacaoyer (Theobroma cacao L.) au Gabon. Revue de Cytologie et Biologies Végétales - Le Botaniste, 29: 13-21.

Engone Obiang NL, Sallé G. 2006. Faut-il éradiquer Phragmanthera capitata, parasite des hévéas en Afrique? $C$. $R$. Biologies, 329: 185-195.

Hawksworth FG, Wiens D. 1996. Dwarf Mistletoes: Biology, Pathology and 
Systematics. Agricultural Handbook 709. US Department of Agriculture: Washington, DC.

Gill LS, Onybe HI. 1990. Mistletoes on rubber trees in Nigeria. Haustorium, 23: $1-2$.

Hévécam. 1995. Rapport de la direction d'exploitation agricole: bilan de l'essai Loranthus (12-AGRO/DEA du 17/02/95), 9p.

Hévécam. 2006. Climatologie. Station Centrale de Nyétté, $5 p$.

Hoffmann AJ, Fuentes ER, Cortès I, Liberona F, Costa V. 1986. Tristerix tetrandrus (Loranthaceae) and its host-plants in the Chilean mattoral: patterns and mechanisms. Oecologia, 69: 202- 206.

Overton JM. 1994. Dispersal and infection Mistletoe metapopulations. Journal of Ecology, 82: 711-723.

Parker C, Riches CR. 1993. Parasitic Weeds of the World: Biology and Control. CAB International Ed.; p.332.
Raynal-Roques A, Paré J. 1998. Biodiversité des Phanérogames parasites: leur place dans la classification systématique. Adansonia, 20: 313-322.

Sallé G. 1994. Rapport de la mission Loranthaceae réalisée au Cameroun, 19p.

Sallé G, Tuquet C, Raynal-Roques A. 1998. Biologie des phanérogames parasites. $C$. R. Soc. Biol., 192: 9-36.

Schall C. 1987. La multiplication de l'avocatier (Persea americana Mill. Cv. Fuerte) par microbouturage in vitro. Fruits, 42(3): 171-176.

Sonké B, Kenfack D, Tindo M. 2000. Parasitisme de l'avocatier (Persea amiricana, Lauraceae) par les Loranthacées dans la région de Yaoundé (Cameroun). Fruits, 55: 325-331.

Zentmyer GA, 1994. Avocado. In Compendium of Tropical Fruit Diseases, Ploetz RC, Zentmyer GA, Nishijima WT, Rohrbach HD (eds). APS Press: EtatsUnis; 71-72. 